\title{
Optimal Discrete-Time Design of Three-Axis Magnetic Attitude Control Laws
}

\author{
Tiziano Pulecchi, Marco Lovera, and Andras Varga
}

\begin{abstract}
The problem of designing discrete-time attitude controllers for three-axis stabilization of magnetically actuated spacecraft is considered. Several methods are discussed and an approach to the tuning of various classes of projection-based controllers is proposed relying on periodic optimal output feedback control techniques. The main advantages of the proposed methods are discussed and illustrated in a simulation study.
\end{abstract}

Index Terms-Attitude control, magnetic actuators, periodic optimal control, periodic systems, spacecraft control, static output feedback.

\section{INTRODUCTION}

$\mathbf{E}$ LECTROMAGNETIC actuators are a particularly effective and reliable technology for the attitude control of small satellites. Such actuators operate on the basis of the interaction between the magnetic field generated by a set of three orthogonal, current-driven coils and the magnetic field of the Earth and provide a simple solution to the problem of generating torques on board of a satellite, both for attitude control in momentum biased or gravity gradient architectures and as secondary actuators for momentum management tasks in zero momentum reaction wheel based configurations.

The main difficulty in the design of magnetic attitude control laws is related to the fact that magnetic torques are instantaneously constrained to lie in the plane orthogonal to the local direction of the geomagnetic field vector. Controllability of the attitude dynamics is ensured for a wide range of orbit altitudes and inclinations in spite of this constraint, thanks to the variability of the geomagnetic field. However, this implies that the attitude control engineer has to deal with a time-varying model in the control design process.

In recent years, considerable effort has been devoted to the analysis of this control problem. In particular, as far as the linear attitude regulation problem is concerned, the following two main lines of work can be identified in the literature.

- Methods based on averaged models. The idea is to replace the time-varying dynamics of the magnetically actuated spacecraft with an approximate time-invariant model obtained using averaging techniques. The advantage of this approach is that the control problem becomes time-invariant. The main drawbacks are that: the designer has to

Manuscript received August 01, 2007; revised January 14, 2009. Manuscript received in final form June 01, 2009. First published August 11, 2009; current version published April 23, 2010. Recommended by Associate Editor S. Kim. This work was supported by the Italian National Research Project "New Techniques of Identification and Adaptive Control for Industrial Systems".

T. Pulecchi and M. Lovera are with the Dipartimento di Elettronica e Informazione, Politecnico di Milano, Milano 20133, Italy (e-mail: pulecchi@elet. polimi.it; lovera@elet.polimi.it).

A. Varga is with the Institute of Robotics and Mechatronics, German Aerospace Center, DLR, D-82234 Wessling, Germany (e-mail: andras.varga@dlr.de).

Digital Object Identifier 10.1109/TCST.2009.2024757 verify a posteriori that the designed controller actually stabilizes the original time-varying dynamics with a satisfactory performance level; the approach is applicable only to configurations for which the averaged model is completely controllable; averaging implies limitations in closed-loop performance. This approach, originally proposed in [1], was further developed in [2] to deal with the (relatively simple) stabilization problem for the coupled roll/yaw dynamics of a momentum biased spacecraft using a magnetic torquer aligned with the pitch axis.

- Methods based on full periodic models. As the variability of the geomagnetic field is almost time-periodic, most of the recent work on the linear magnetic attitude control problem has focused on the use of optimal and robust periodic control theory for the design of state and output feedback regulators [3]-[11]. While periodic control design methods have the advantage of guaranteeing closed-loop stability a priori, they lead to the synthesis of time-periodic regulators, which are difficult to implement and operate in practice for a number of reasons (see Section III-A). At the present stage, the only attempt at designing a constant gain periodically optimal controller (see [8]) has led to a design procedure which still calls for a posteriori stability testing.

In light of the above discussion, the aim of this brief is to present the results obtained in the application of periodic LQ optimal control techniques to the design of digital attitude controllers for spacecraft equipped with magnetic actuators. The proposed approach allows the design of periodic control laws parameterized via constant gains with guaranteed nominal stability and LQ performance, using an optimization-based method. More precisely, we will focus on the optimal tuning of the so-called "projection-based" controllers for magnetic attitude regulation (first proposed in [12]), a controller structure widely used in the practice of magnetic attitude control.

Note that the magnetic attitude control design problems associated with periodic optimization techniques pose a significant challenge from the numerical point of view. Indeed the open-loop attitude dynamics is usually marginally stable (i.e., undamped) or unstable and the time-variability of the dynamics introduced by the magnetic actuators is characterized by a very long period. In particular, the design problems discussed in the brief were solved by using reliable numerical methods such as those implemented in the Periodic Systems Toolbox for MATLAB developed by one of the authors (see, e.g., [13]).

This brief is organized as follows. Section II provides a description of the spacecraft considered in the study as well as the derivation of a linearized model for its attitude dynamics. The considered control design techniques are subsequently described in Section III, while the results obtained in the simulation of the designed control laws are presented and discussed in Section IV. 
Notation: For a square time-varying matrix $A(k)$, $k=0,1, \ldots$ we denote $\Phi_{A}(j, i)=A(j-1) A(j-2), \ldots, A(i)$ for $j>i$ and $\Phi_{A}(i, i)=I$. If $A(k)$ is periodic with period $N$, the matrix $\Phi_{A}(\tau+N, \tau)$ is called the monodromy matrix of system $x(k+1)=A(k) x(k)$ at time $\tau$ and its eigenvalues, independent of $\tau$, are called characteristic multipliers. Finally, $I_{n}$ denotes the $n \times n$ identity matrix and $O_{m \times n}$ a $m \times n$ matrix with null elements.

\section{LINEARIZED ATTITUDE DYNAMICS}

In order to represent the attitude motion of an Earth-pointing spacecraft on a circular orbit the following reference systems are adopted.

- Earth Centered Inertial Reference Axes (ECI). The Earth's center is the origin of these axes. The positive $X$-axis points in the vernal equinox direction. The $Z$-axis points in the direction of the North Pole. The $Y$-axis completes the right-handed orthogonal triad.

- Orbital Axes $\left(X_{0}, Y_{0}, Z_{0}\right)$. The origin of these axes is at the satellite centre of mass. The $X$-axis points to the Earth's centre; the $Y$-axis points in the direction of the orbital velocity vector. The $Z$-axis is normal to the satellite orbit plane.

- Satellite Body Axes. The origin of these axes is at the satellite center of mass; in nominal Earth-pointing conditions the $X_{b}$ (yaw), $Y_{b}$ (roll), and $Z_{b}$ (pitch) axes are aligned with the corresponding orbital axes.

We will consider a spacecraft with inertia matrix $I=$ $\operatorname{diag}\left[\begin{array}{lll}I_{x x} & I_{y y} & I_{z z}\end{array}\right]$, equipped with a single momentum wheel aligned with the body $z$ axis, with moment of inertia $J$ and angular velocity $\Omega$ relative to the body frame. The aim of the attitude control scheme is to maintain the satellite body axes aligned with the orbital axes, while exploiting the gyroscopic effect due to the momentum wheel. Note that the choice of this spacecraft configuration is not particularly restrictive, since by assuming that $\Omega=0$ and $I_{x x} \ll I_{y y}, I_{x x} \ll I_{z z}$ a gravity-gradient configuration can be obtained as a particular case. Similarly, the assumptions of a circular orbit and of a diagonal inertia matrix are made only for ease of presentation, but they are by no means necessary for the applicability of the proposed design approach. Indeed, unlike existing design methods based on averaging (see, e.g., [2]), inertial coupling between roll/yaw and pitch dynamics can be handled in the design problem. The angular kinematics and dynamics of the spacecraft are modelled using as state variables the quaternion $q=\left[\begin{array}{ll}q_{1} & q_{R}^{T}\end{array}\right]^{T}=\left[\begin{array}{llll}q_{1} & q_{2} & q_{3} & q_{4}\end{array}\right]^{T}$ describing the attitude of the body axes with respect to the orbital axes, and the inertial angular velocity vector $\omega=\left[\begin{array}{lll}\omega_{x} & \omega_{y} & \omega_{z}\end{array}\right]^{T}$, with respect to the body axes.

In the following we will derive linearized dynamic models for the formulation of this control problem (note, in passing, that the use of a linearized model is fully justified in most applications by specifications requiring pointing accuracies of at most a few degrees and pointing stabilities of fractions of the orbital angular rate). With respect to the selected state variables, the nominal, Earth-pointing, equilibrium corresponds to the attitude quaternion $\bar{q}=\left[\begin{array}{cccc}1 & 0 & 0 & 0\end{array}\right]^{T}$ and to the angular rate $\bar{\omega}=\left[\begin{array}{cc}0 & 0-\Omega_{0}\end{array}\right]^{T}$, where $\Omega_{0}$ is the orbital angular rate.
Define the state vector $x_{c}=\left[\begin{array}{ll}\delta q_{R}^{T} & \delta \omega^{T}\end{array}\right]^{T}$ formed with small displacements of the vector part $q_{R}$ of the attitude quaternion $q$ from the nominal values $\bar{q}_{R}=\left[\begin{array}{ccc}0 & 0 & 0\end{array}\right]^{T}$ and small deviations of the body rates from the nominal values $\bar{\omega}_{x}=\bar{\omega}_{y}=$ $0, \bar{\omega}_{z}=-\Omega_{0}$. Then the attitude dynamics can be linearized and the local linear dynamics for the attitude can be defined as [6]

$$
\dot{x}_{c}(t)=A_{c} x_{c}(t)+B_{c T}\left[T_{c, \text { mag }}(t)+T_{c, \text { dist }}(t)\right],
$$

where $T_{c, \text { mag }}$ is the magnetic control torque vector and $T_{c, \text { dist }}$ is the disturbance torque vector. Taking into account that $T_{c, \mathrm{mag}}$ can be written as

$$
T_{c, \operatorname{mag}}(t)=m_{c}(t) \times b(t)=S(b(t)) m_{c}(t)=-b(t) \times m_{c}(t)
$$

where $b=\left[\begin{array}{lll}b_{x} & b_{y} & b_{z}\end{array}\right]^{T}$ is the geomagnetic field vector (in body frame), $m_{c}$ is the dipole vector of the magnetic torquers and

$$
S(b)=\left[\begin{array}{ccc}
0 & b_{z} & -b_{y} \\
-b_{z} & 0 & b_{x} \\
b_{y} & -b_{x} & 0
\end{array}\right] .
$$

Equation (1) can be equivalently written as

$$
\dot{x}_{c}(t)=A_{c} x_{c}(t)+B_{c m}(t) m_{c}(t)+B_{c T} T_{c, \text { dist }}(t)
$$

where

$$
\begin{aligned}
A_{c} & =\left[\begin{array}{ccc|ccc}
0 & -\Omega_{0} & 0 & 0.5 & 0 & 0 \\
\Omega_{0} & 0 & 0 & 0 & 0.5 & 0 \\
0 & 0 & 0 & 0 & 0 & 0.5 \\
\hline 0 & 0 & 0 & 0 & W_{x} & 0 \\
0 & -6 k_{y} \Omega_{0}^{2} & 0 & W_{y} & 0 & 0 \\
0 & 0 & +6 k_{z} \Omega_{0}^{2} & 0 & 0 & 0
\end{array}\right] \\
B_{c T} & =\left[\begin{array}{ccc}
0 & 0 & 0 \\
0 & 0 & 0 \\
0 & 0 & 0 \\
I_{x x}^{-1} & 0 & 0 \\
0 & I_{y y}^{-1} & 0 \\
0 & 0 & I_{z z}^{-1}
\end{array}\right], B_{c m}(t)=B_{c T} S(b(t))
\end{aligned}
$$

and $k_{x}=I_{y y}-I_{z z} / I_{x x}, k_{y}=I_{z z}-I_{x x} / I_{y y}, k_{z}=$ $I_{x x}-I_{y y} / I_{z z}, W_{x}=-k_{x} \Omega_{0}+k_{w x} \bar{\Omega}, W_{y}=-k_{y} \Omega_{0}-k_{w y} \bar{\Omega}$ $k_{w x}=J / I_{x x}, k_{w y}=J / I_{y y}$. Here, $\bar{\Omega}$ is the nominal wheel speed.

Note that two different control matrices $B_{c T}$ and $B_{c m}(t)$ have been defined, in order to handle problem formulations in which either magnetic torques $\left(T_{c, \mathrm{mag}}\right)$ or magnetic dipoles $\left(m_{c}\right)$ are used as control variables, respectively. In any case, $B_{c T}$ also describes the effect of disturbance torques on the linearized attitude dynamics. Note that, while $A_{c}$ is constant, the control matrix $B_{c m}(t)$ corresponding to the control input $m_{c}$ turns out to be time-varying because of the dependence on the geomagnetic field vector $b(t)$. Such time-variability turns out to be approximately time-periodic with period equal to the orbital period $\tau_{0}=2 \pi / \Omega_{0}$. Deviations from exact periodicity are due to Earth rotation and to orbit perturbations.

Remark 1: As can be seen from equation (4) (and well known in the literature, see, e.g., [14], [15]), the $A_{c}$ matrix shows that 
the pitch dynamics is decoupled from the roll/yaw dynamics. Therefore, in the case of a spacecraft equipped with three independent torque actuators it would be possible to design separate controllers for the two subsystems. Unfortunately, as can be seen from the expression of matrix $B_{c m}(t)$ in (5), the use of magnetic actuators introduces a coupling between the roll/yaw and the pitch subsystems. Similarly, coupling terms appear if the spacecraft inertia matrix $I$ is not diagonal-a situation which occurs frequently in practice.

Finally, since we are concerned with a discrete-time design problem, suitable discrete-time equivalents of (1) and (3) have been derived, in the forms

$$
\begin{aligned}
& x(k+1)=A x(k)+B_{T}\left[T_{\mathrm{mag}}(k)+T_{\text {dist }}(k)\right] \\
& x(k+1)=A x(k)+B_{m}(k) m(k)+B_{T} T_{\text {dist }}(k)
\end{aligned}
$$

respectively, where for a sampling-time of $\Delta=2 \pi /\left(N \Omega_{0}\right)(N$ is the discrete-time period, i.e., the number of samples in one orbital period $\tau_{0}$ ) we have

$$
\begin{aligned}
A & :=\exp \left(A_{c} \Delta\right) \\
B_{T} & :=\int_{0}^{\Delta} e^{A_{c}(\Delta-\tau)} B_{c T} d \tau, \\
B_{m}(k) & :=\int_{k \Delta}^{(k+1) \Delta} e^{A_{c}[(k+1) \Delta-\tau]} B_{c m}(\tau) d \tau \\
x(k) & :=x_{c}(k \Delta) \\
T_{\mathrm{mag}}(k) & :=T_{c, \text { mag }}(k \Delta), \\
T_{\text {dist }}(k) & :=T_{c, \text { dist }}(k \Delta), \quad m(k):=m_{c}(k \Delta) .
\end{aligned}
$$

\section{Controller Design}

In the following, the problem of designing a suitable controller for the linearized models (6) and (7) is considered. In particular, Section III-A provides some background on optimal periodic control theory and a discussion of the pros and cons of this approach to the magnetic attitude control problem; the class of projection-based magnetic attitude controllers is presented in Section III-B, while the proposed approach to the design of such controllers is dealt with in Section III-C.

\section{A. Periodic Optimal State Feedback Controller}

A significant portion of the recent literature on magnetic attitude control attempted to solve the problem by resorting to optimal periodic control theory (see, e.g., [3], [5], [6], [8], and [16]). A brief overview of optimal periodic control (in discretetime) is provided in this section in order to highlight the advantages and disadvantages of this approach to the considered problem.

Consider the linear discrete-time periodic system

$$
\begin{aligned}
x(k+1) & =A(k) x(k)+B(k) u(k) \\
y(k) & =C(k) x(k)
\end{aligned}
$$

and the linear-quadratic (LQ) criterion $\sum_{k=0}^{\infty}\left[x^{T}(k) Q(k) x(k)+u^{T}(k) R(k) u(k)\right]$, $Q(k) \geq 0, R(k)>0$ are symmetric $N$-periodic matrices. Under suitable assumptions (see [17]), the optimal $N$-periodic state-feedback matrix $F(k)$ minimizing the performance index $J$ is given by $F(k)=$ $-\left(R(k)+B^{T}(k) X(k+1) B(k)\right)^{-1} B^{T}(k) X(k+1) A(k)$, where the $N$-periodic symmetric positive semi-definite matrix $X(k)$ satisfies the reverse discrete-time periodic Riccati equation $X(k)=Q(k)+A^{T}(k) X(k+1) A(k)-A^{T}(k) X(k+$ 1) $B(k)\left(R(k)+B^{T}(k) X(k+1) B(k)\right)^{-1} B^{T}(k) X(k+1) A(k)$.

The optimal periodic LQ approach has the obvious advantage of providing a stabilizing controller with a guaranteed level of performance, as expressed by the minimization of the cost function $J$. The issues associated with the implementation of optimal periodic controllers, however, make their actual application in real satellite missions not very likely. The main difficulties are related with the storage requirements for a fully time-periodic gain, the accurate time-synchronization of the time-varying controller and finally its robustness to variations in the time-history of the geomagnetic field due to orbit precession. While these issues motivate the interest in alternative approaches to this design problem, the performance level provided by the optimal periodic LQ controller can be taken as a reference for all other design methods.

\section{B. Fixed-Gain Projection-Based Controllers}

A very common structure for magnetic attitude control laws, which goes back to classical papers such as [12], consists of discrete-time control laws of the kind

$$
u(k)=m(k)=\frac{1}{\|b(k \Delta)\|^{2}} S^{T}(b(k \Delta)) T_{i d}(k)
$$

where $b(k \Delta)$ is the measurement, at discrete time $k$, of the geomagnetic field $b$ and $T_{i d}(k)$ is an "ideal" control torque to be determined on the basis of a suitable feedback of state or output variables, according to the specific attitude control architecture of the considered spacecraft. The above control law can be readily given a simple geometric interpretation. Indeed, assume for a moment that (14) can be implemented in continuous time; then, recalling (2) we can express the torque generated by the magnetic coils as

$$
T_{c, \operatorname{mag}}(t)=\frac{1}{\|b(t)\|^{2}} S(b(t)) S^{T}(b(t)) T_{i d}(t)
$$

which can be easily interpreted as the projection of vector $T_{i d}$ onto the plane orthogonal to the direction of the magnetic field vector $b$ (hence the name of "projection-based" controllers). In view of the digital implementation of the controller, this geometric view of magnetic control holds only in an approximate sense, but still represents a good interpretation of the operation of the controller since the sampling interval $\Delta$ is normally small (from one second to a few dozens of seconds at most) with respect to the period of the geomagnetic field along a low Earth orbit (the orbital period of a LEO orbit is typically about $6000 \mathrm{~s}$ ). Some examples of possible controller structures corresponding to (14) are discussed in Section IV-C. Note that the advantage of the considered controller structure is that only constant parameters (i.e., the ones defining $T_{i d}$ as a function of the state or output vector) have to be designed, while the time-dependence 
of the control law is carried by the (measurable) value of the geomagnetic field $b$ entering (14). However, to the best of our knowledge, no systematic design approaches to the selection of the parameters in the proposed control laws (14) for a three-axis magnetically stabilzed spacecraft are available. In Section III-C, an approach to the optimal tuning of this class of attitude controllers will be proposed.

\section{Optimal Periodic Design of Fixed-Gain Projection-Based Controllers}

In this brief, we propose an approach to the tuning of projection-based controllers based on numerical techniques for the solution of optimal periodic output feedback control problems [4]. This approach relies on a gradient-based optimization method to determine time-periodic output feedback controllers by minimizing a suitable quadratic cost function. In this section, an overview of this technique will be presented.

Consider the system (12)-(13). The design problem entails minimizing the LQ criterion

$$
J=E\left\{\sum_{k=0}^{\infty}\left[x^{T}(k) Q(k) x(k)+u^{T}(k) R(k) u(k)\right]\right\}
$$

where $Q(k) \geq 0, R(k)>0$ are symmetric $N$-periodic matrices, in order to determine the optimal stabilizing constant gain output feedback controller, of the form

$$
u(k)=F y(k)=F C(k) x(k) .
$$

Note that (16) differs from the LQ criterion introduced in Section III-A because of the expectation operator. It is important to point out that the expectation is taken over the set of initial states (and has nothing to do with time averaging as introduced in other design approaches for magnetic attitude control, see, e.g., [2]). Indeed, in order to use the performance criterion to find an optimal feedback gain $F$ it is necessary to eliminate the dependence on $x_{0}$. More precisely, it will be assumed in the following that the initial state $x_{0}$ is a random variable with zero mean and covariance $X_{0}=E\left[x_{0} x_{0}^{T}\right]$.

The proposed approach for the solution of this time-periodic design problem is based on the application of gradient-based optimization techniques which exploit the possibility of efficiently computing both the cost function (16) and its gradient with respect to the parameters of the considered controller (17). The expressions of function and gradient can be computed on the basis of the following result (see [4] for details and proofs).

Proposition 1: Let $F$ be a constant stabilizing output feedback matrix and denote $\bar{A}(k)=A(k)+B(k) F C(k)$ and $\bar{Q}(k)=Q(k)+C^{T}(k) F^{T} R(k) F C(k)$. Then the expressions for the cost function (16) and its gradient with respect to the elements of $F$ are given by

$$
\begin{aligned}
J(F)= & \operatorname{tr}\left(P(0) X_{0}\right) \\
\nabla_{F} J(F)= & 2 \sum_{j=0}^{N-1}\left(R(j) F C(j)+B^{T}(j) P(j+1) \bar{A}(j)\right) \\
& \times S(j) C^{T}(j)
\end{aligned}
$$

where $P(k)$ and $S(k)$ satisfy, respectively, the discrete periodic Lyapunov equations (DPLEs)

$$
\begin{aligned}
P(k) & =\bar{A}^{T}(k) P(k+1) \bar{A}(k)+Q(k), \\
P(N) & =P(0) \\
S(k+1) & =\bar{A}(k) S(k) \bar{A}^{T}(k)+G(k), \\
S(N) & =S(0)
\end{aligned}
$$

with

$$
G(k)= \begin{cases}0, & k \neq N-1 \\ X_{0}, & k=N-1\end{cases}
$$

From a numerical point of view, each evaluation of the performance index (16) and its gradient involves (see Proposition 1) the solution of a pair of DPLEs: a reverse time DPLE and a dual forward time DPLE, where it is assumed that all characteristic multipliers of the monodromy matrix of the closed loop system lie inside the open unit circle of the complex plane. This ensures existence of a unique solution of both equations. Note that for standard systems these are two discrete Lyapunov equations, which can be solved efficiently with a computational cost marginally greater than the cost of solving a single Lyapunov equation. The preservation of this feature is even more desirable for the periodic case, because of the much higher computational effort involved in solving a single periodic Lyapunov equation. Fortunately, this goal can be achieved with the recently proposed numerically stable algorithms to solve DPLEs [18]. The optimal tuning of the proposed control law (14) can be determined using a suitable function available in the Periodic Systems Toolbox [13]. This function is based on a gradient-based function minimization technique for problems with simple bounds (limited memory Broyden-Fletcher-Goldfarb-Shanno-BFGS method). To achieve the highest efficiency, the function and gradient evaluations have been implemented as a Fortran 95 mex-function based on the above results.

Remark 2: It is important to note that the application of Proposition 1 to control design problems requires a way of obtaining an initial stabilizing gain, in order to reduce the numerical difficulties associated with open loop unstable dynamics, and to facilitate convergence of the iterative optimization procedure. In the cases of constant-gain state feedback and dynamic output feedback, the initial gain can be selected according to the guidelines provided by [19, Prop. 1], [20] for the globally stabilizing tuning of state feedback magnetic attitude controllers of the projection-based type [i.e., equation (14)]. Indeed, using the cited result it is possible to show that for the control law

$$
T_{i d}=-\left(\varepsilon^{2} k_{p} \delta q_{R}+\varepsilon k_{d} I \delta \omega\right)
$$

there exist positive scalars $\varepsilon^{\star}, k_{p}$, and $k_{d}$ such that for any $0<\varepsilon<\varepsilon^{\star}(22)$ ensures exponential stability for the closed loop system. Therefore, it is possible to obtain an initial stabilizing controller with the structure given in (22) by first choosing the positive gains $k_{p}, k_{d}$ and an initial value of $\varepsilon$ and subsequently reducing $\varepsilon$ until stability is achieved. Finally, in the output feedback case one can follow the general approach outlined in [4] for the initialization of the optimization process. Note that the 
control law (22) will only be considered for initialization purposes and will not be actually implemented on the spacecraft.

Remark 3: Once a stabilizing initial gain has been obtained, the gradient-based iterative procedure can be used in order to numerically optimize the cost function (16) [or, equivalently, (18)] using the gradient information provided by (19). It is important to point out that such an iterative procedure can guarantee that closed-loop stability is intrinsically maintained from one iteration to the following one as the gradient of the cost function is computed on the basis of the Lyapunov equation (20), which implicitly enforces stability of the closed-loop system for the current value of the controller gain.

Remark 4: Recently, the feasibility of techniques based on linear and bilinear matrix inequalities (LMI, BMI) for the design of periodic controllers has been explored [21]. While this approach lends itself to the formulation of more general control problems, it suffers from a significant drawback, i.e., it is limited to relatively small scale problems (both in terms of order and period) when compared to techniques relying on the solution of periodic Lyapunov and Riccati equations.

\section{Simulation Study}

In this section, the performance of the considered control laws will be discussed in a detailed simulation study, and the results will be compared to those provided by the reference optimal periodic state feedback (PSF) LQ control strategy. For all the control laws, two values for the number of sampling points $N$ over one orbit have been considered, namely $N=100$ and $N=300$, corresponding respectively to a sampling interval $\Delta$ of about 56.1 and $18.7 \mathrm{~s}$.

\section{A. Spacecraft Parameters}

The considered spacecraft is of the type described in Section II; the numerical values for the parameters of the linearized model are as follows:

- satellite inertia $\left(\mathrm{kg} \mathrm{m}^{2}\right): I=\operatorname{diag}\left[\begin{array}{lll}35 & 17 & 25\end{array}\right]$;

- momentum wheel inertia $\left(\mathrm{kg} \mathrm{m}^{2}\right): J=0.01$;

- nominal wheel speed $(\mathrm{rad} / \mathrm{s}): \bar{\Omega}=-200$.

A saturation limit of $\pm 20 \mathrm{Am}^{2}$ for the magnetic coils has been assumed. The considered configuration corresponds to a satellite characterized by coupled roll/yaw dynamics which is marginally stable thanks to the wheel momentum, and unstable pitch dynamics, due to the unfavorable gravity gradient effect $\left[k_{z}>0\right.$ in (4)]. Note, however, that the open loop instability of the pitch dynamics is compensated by the fact that the pitch axis is easier to control using magnetic torquers than the roll and yaw axes, as for the considered (polar) orbit the geomagnetic field lies essentially in the orbital plane, so that the pitch dynamics is controllable throughout the entire orbit.

The spacecraft operates in a near polar orbit ( $87^{\circ}$ inclination) with an altitude of $450 \mathrm{~km}$ and a corresponding orbital period of $5614.8 \mathrm{~s}$. For this orbit, the following nominal (periodic) model for the components of the geomagnetic field has been considered (Tesla):

$$
b(t)=10^{-6}\left[\begin{array}{c}
7 \cos \left(\Omega_{0} t\right)+48 \sin \left(\Omega_{0} t\right) \\
23 \cos \left(\Omega_{0} t\right)-2 \sin \left(\Omega_{0} t\right) \\
5
\end{array}\right] .
$$

It is important to point out that the above nominal periodic representation for the geomagnetic field has been used only as a design model. A detailed, highly accurate model of the geomagnetic field has been used in the simulation study (see Section IV-B) in order to validate the performance of the designed controllers.

\section{B. Simulation Environment}

The simulations presented in the following have been carried out using an object-oriented environment for satellite dynamics (see [22] for details) developed using the Modelica language [23]. More precisely, a full nonlinear simulation of the coupled rigid body orbital and attitude dynamics has been performed and the following models of the space environment have been implemented: the JGM-3 spherical expansion for the geopotential as a gravitational model, the Harris-Priester model for the atmospheric density distribution (see [24] for details) and the International Geomagnetic Reference Field (IGRF, see [25]) for the Earth's magnetic field (up to order 10). Disturbance torques due to gravity gradient (including $J_{2}$ effects), magnetic residual dipole (assuming a residual dipole of $1 \mathrm{Am}^{2}$ along each spacecraft body axis) and solar radiation pressure (computed using the solar coordinates formulas given in [24]) have been taken into account in the simulation. Finally, all the controllers have been implemented in digital form, using a conventional sample and hold scheme.

\section{Controller Tuning}

Different control strategies, using the fixed structure projection approach, were adopted, namely as follows.

- Constant-Gain State Feedback Control (CSF): $T_{i d}(k)=$ $K_{x} x(k)$.

- Constant-Gain Output Feedback Control (COF): $T_{i d}(k)=K_{y} y(k), y(k)=C(k) x(k)$. In particular, the case of output consisting of measured angular rates, and pitch and roll angles (quaternion components $q_{3}$ and $q_{4}$ ) only is considered.

- Dynamic output feedback control, where the output is assumed to consist of the measured quaternion and a reconstructed angular rate vector. Two design approaches have been analyzed: a kinematic reconstruction of the angular rate vector alone (DOF1) and an online estimation of the whole system state via Kalman filtering (DOF2). In the former case, system (3) has been augmented with a pseudo-derivative filter with a time constant of $T_{p d}$ seconds (set to $T_{p d}=10 \Delta$ )

$$
\begin{aligned}
T_{p d} \dot{z} & =-z+\delta q_{R} \\
\delta \hat{\dot{q}}_{R} & =\frac{1}{T_{p d}}\left(-z+\delta q_{R}\right)
\end{aligned}
$$

which, recalling (3) and (4), can be used to compute an estimate of the spacecraft angular rate as

$$
\begin{aligned}
\delta \hat{\omega} & =A_{c 12}^{-1}\left(\delta \hat{\dot{q}}_{R}-A_{c 11} \delta q_{R}\right) \\
& =2\left(\frac{1}{T_{p d}}\left(\delta q_{R}-z\right)-A_{c 11} \delta q_{R}\right) .
\end{aligned}
$$


TABLE I

OPEN- AND CLOSED-LOOP STABILITY DEgREE AND COST FunCTION FOR $N=100$ AND $N=300$ FOR THE CONSIDERED CONTROL DESIGN METHODS

\begin{tabular}{ccccc} 
& $\rho(N=100)$ & $J_{\text {opt }}(N=100)$ & $\rho(N=300)$ & $J_{\text {opt }}(N=300)$ \\
\hline open loop & $1.0243 \mathrm{e}+004$ & & $1.0243 \mathrm{e}+004$ & \\
$P S F$ & $2.2812 \mathrm{e}-002$ & $5.89 \mathrm{e}+001$ & $1.017 \mathrm{e}-004$ & $1.3162 \mathrm{e}+002$ \\
$C S F$ & $5.3867 \mathrm{e}-002$ & $6.441 \mathrm{e}+001$ & $3.889 \mathrm{e}-003$ & $1.3830 \mathrm{e}+002$ \\
$C O F$ & $5.9256 \mathrm{e}-002$ & $7.6545 \mathrm{e}+001$ & $2.3771 \mathrm{e}-003$ & $1.6847 \mathrm{e}+002$ \\
$D O F 1$ & $2.0356 \mathrm{e}-002$ & $4.2717 \mathrm{e}+002$ & $2.1510 \mathrm{e}-002$ & $5.1820 \mathrm{e}+002$ \\
$D O F 2$ & $5.3867 \mathrm{e}-002$ & $6.441 \mathrm{e}+001$ & $3.889 \mathrm{e}-003$ & $1.3830 \mathrm{e}+002$ \\
\hline
\end{tabular}

The augmented system given by (3) and (23)-(24) is therefore given by

$$
\begin{aligned}
x_{a} & =\left[\begin{array}{lll}
\delta q_{R}^{T} & \delta \omega^{T} & z^{T}
\end{array}\right]^{T} \\
\dot{x_{a}} & =\left[\begin{array}{ccc} 
& A_{c} & O_{6 \times 3} \\
\frac{1}{T_{p d}} I_{3} & O_{3} & -\frac{1}{T_{p d}} I_{3}
\end{array}\right] x_{a}+\left[\begin{array}{c}
B_{c T} \\
O_{3 \times 6}
\end{array}\right] u .
\end{aligned}
$$

In the latter case, the system state (attitude and angular rates) is estimated online via a linear time-invariant Kalman filter processing the satellite's attitude measured data. The state and output covariance matrices in the design of the filter have been chosen respectively as $Q_{e}=\left(3 \times 10^{-3}\right)^{2} I_{6}$ and $R_{e}=\operatorname{diag}\left[\begin{array}{lll}4 \times 10^{-4} & 4 \times 10^{-4} & 10^{-2}\end{array}\right]$ (corresponding to typical values of error variance for star tracker measurements).

All the proposed control laws have been designed using the Periodic Systems Toolbox for MATLAB. The weighting matrices in the quadratic cost function (16) have been chosen as $Q=$ $0.01 I_{n}$ and $R=100 I_{3}$, where $n$ is the state dimension.

The results obtained for the proposed controller designs are presented in Table I and can be summarized as follows.

- Closed Loop Stability: Since we are dealing with a linear time-periodic system, closed loop stability must be checked using Floquet theory (see [17]). This requires to check if the eigenvalues of the closed loop monodromy matrix $\Phi_{\bar{A}}(N, 0)$ (i.e., the transition matrix for the closed-loop system over one period) are less than unity in modulus. In the first and third columns of Table I the obtained values for the spectral radius $\rho=\max _{1<i<n}\left(\left|\lambda_{i}\right|\right)$ of the monodromy matrix are shown, for $N \stackrel{1 \leq i \leq n}{=} 100$ and $N=300$, respectively. As can be inferred from the Table all the designed controllers lead to asymptotically stable closed loop dynamics, i.e., to a spectral radius smaller than one.

- Optimality: The results obtained using periodic optimal state feedback control (PSF) and the fixed structure controllers can be compared directly in terms of the achieved optimal values of the cost function (16) (second and fourth columns of Table I, for $N=100$ and $N=300$, respectively). The performance loss associated with the adoption of the fixed structure controller instead of the optimal periodic one turns out to be acceptable for the constant state feedback, COF and DOF2 design cases, both for $N=100$ and $N=300$. On the other hand, the DOF1 designs do not seem to be able to cope with the LQ-optimal reference design level of performance in terms of cost function, while they provide a satisfactory stability degree.
As for the dynamic controllers DOF1 and DOF2, it is apparent from the results summarized in Table I that the use of a Kalman filter ensures far better performance than does a simple pseudo-differentiator for the estimation of the unmeasured angular rate. The DOF2 design provides highly satisfactory performance in terms of both stability degree and cost function.

- Robustness: In order to assess in a quantitative way how stability and performance are affected by uncertainty in the design model, a Monte Carlo simulation approach has been adopted. More precisely, two sets of experiments have been performed, aiming at checking the behavior of the closed-loop system: the principal inertias of the satellite have been perturbed by $\pm 10 \%$; the orbit altitude has been perturbed by $\pm 100 \mathrm{~km}$. For each of the two situations 200 random perturbations have been generated and the values of the stability degree and of the optimal cost have been computed. For the CSF controller the closed-loop system remains stable in all cases, with perturbations on the inertia causing a relatively small effect on the optimal cost and on the stability degree (worst case of 0.13). The perturbation in the orbit altitude, on the other hand, affects the closed-loop system in a more significant way (worst case stability degree of 0.25 ) as it has a strong influence on the strength of the geomagnetic field. It should be kept in mind, however, that a $\pm 100 \mathrm{~km}$ variation in orbit altitude is a rather extreme perturbation. Similar results (omitted for brevity) have been obtained for the other control strategies considered in this brief.

\section{Simulation Results}

In order to illustrate the time domain behavior of the fixed structure controllers, some simulation examples are presented, showing the transient following an initial perturbation of the attitude dynamics with respect to the nominal Earth-pointing equilibrium. In particular, the following initial perturbations have been applied to the angular rate vector: $\delta \omega(0)=\left[\begin{array}{lll}1 \times 10^{-3} & 2 \times 10^{-3} & 0\end{array}\right]^{T} \mathrm{rad} / \mathrm{s}$. While this may appear to be an extremely small initial condition, it actually represents a significant perturbation as far as nominal regulation of attitude dynamics is concerned. For the sake of conciseness, only simulation results for the CSF and COF controllers with $N=100$ are presented (the time histories obtained by implementing the DOF2 controller essentially coincide with the ones for CSF) in Fig. 1.

As can be seen from Fig. 1, both controllers damp out the effect of the initial angular rate perturbation in less than one orbit (an adequate performance for a magnetic attitude control scheme) and bring the system to its steady state response under the effect of the cyclic external disturbance torques. As expected, the yaw and roll axes ( $q_{2}$ and $q_{3}$ components of the attitude quaternion) are strongly coupled and their transients correspond to the response of the stabilized precession and nutation modes of the open-loop dynamics, while the pitch axis ( $q_{4}$ component of the attitude quaternion) is stabilized much more efficiently thanks to the above mentioned favorable controllability properties. Actually, the closed-loop transient associated with the pitch dynamics is too short to be visible on a time scale 

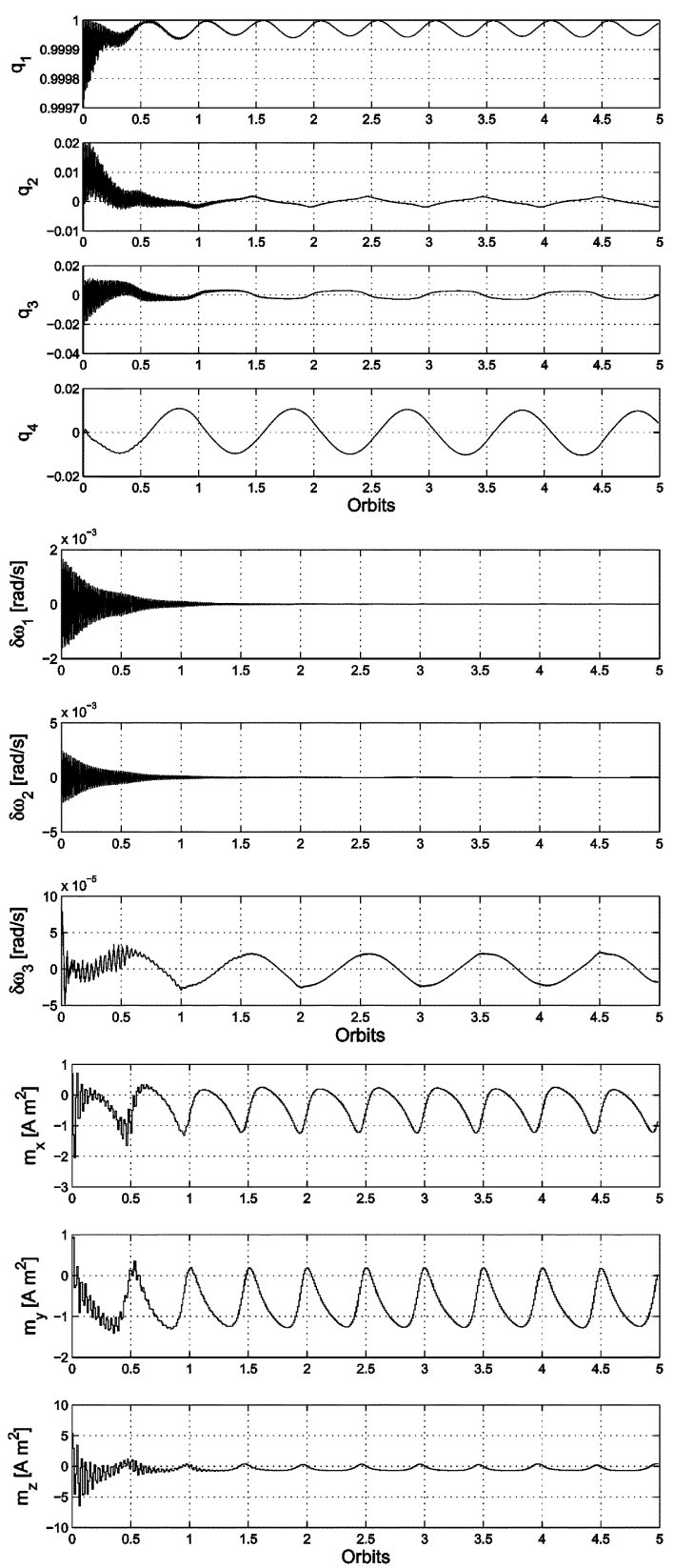

(a)
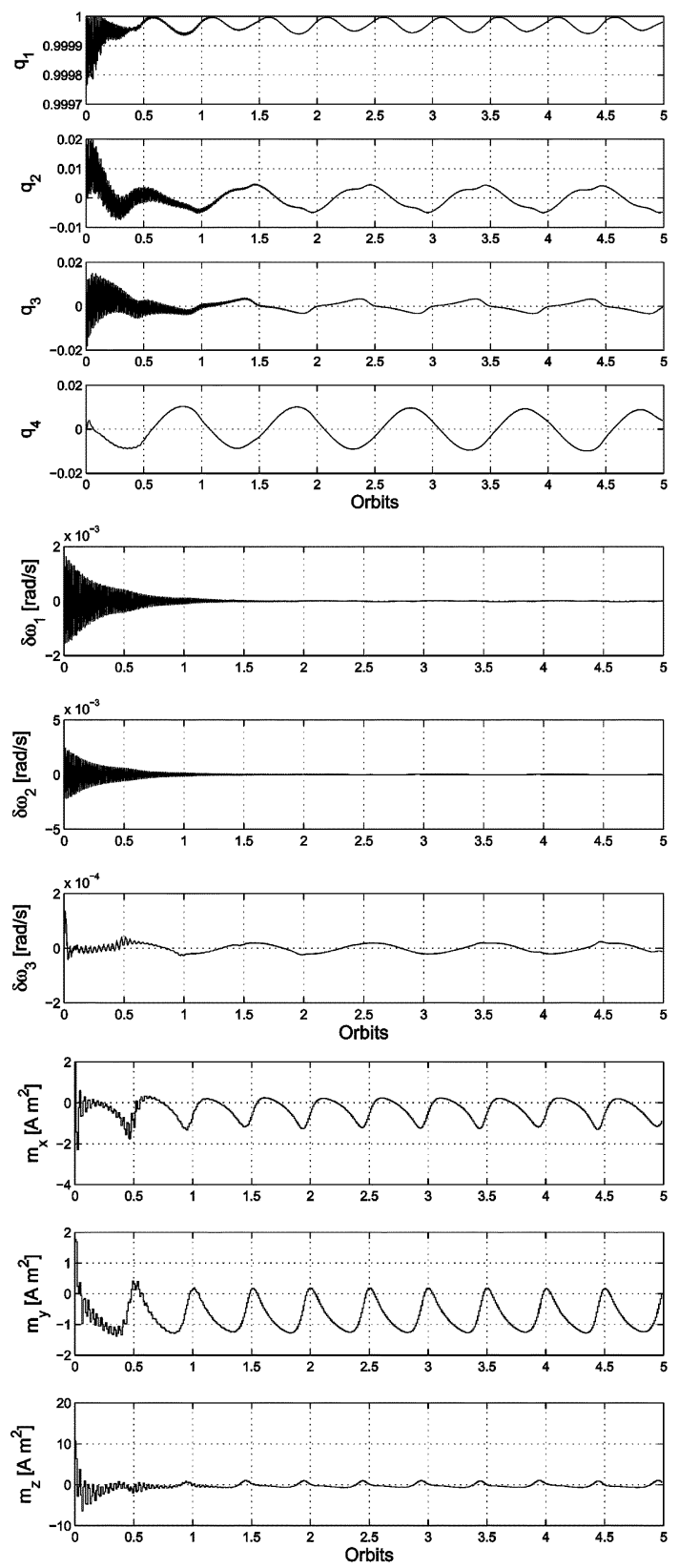

(b)

Fig. 1. Quaternion, angular rates and control dipole moments. Left: constant-gain state feedback controller (CSF) $(N=100)$. Right: constant-gain output feedback controller $\operatorname{COF}(N=100)$.

of five orbits: the oscillation of $q_{4}$ is actually the steady-state response to the external disturbance torques acting on the satellite (see Section IV-B).

In terms of performance, recalling that under small angles assumptions $q_{2}, q_{3}$, and $q_{4}$ correspond to half of the roll, yaw, and pitch angles, respectively, the following conclusions may be drawn. The CSF controller can maintain the angular deviations from the nominal attitude within approximately less than $1^{\circ}$ on all axes. This performance level is normally considered satisfactory for this type of attitude control architecture; note, however, that it has been obtained with fairly coarse sampling and that no attempt has been made at selecting the weights in the cost function (16) in order to optimize the closed-loop performance. The $\mathrm{COF}$ controller is capable of providing the same level of perfor- mance as the CSF about the roll and pitch axes, but suffers from the unavailability of the direct measurement of the yaw angle, as visible from the slightly larger oscillation of $q_{2}$ in Fig. 1. Finally, though figures could not be included due to space limitations, it is interesting to note that the DOF1 controller, which has been tuned using the same choice of weights used in the two previous cases, gives rise to a significantly shorter transient at the beginning of the simulation (the angular rates are damped out in less than half an orbit), but to a less satisfactory steady-state performance (as expected, given the relatively larger value of the optimal cost, see Table I). The faster closed-loop dynamics is achieved at the expense of a larger activation level of the magnetic coils with respect to the CSF and COF controllers (note, however, that all the controllers use only a fraction of the avail- 
TABLE II

OPEn- AND Closed-Loop Stability DegRee AND Cost Function For $Q=0.01 I_{n}$ AND $Q=0.1 I_{n}$ FOR THE CONTROL DESIGN METHODS CONSIDERED IN THE COMPARISON

\begin{tabular}{ccccc} 
& $\rho\left(Q=0.01 I_{n}\right)$ & $J_{\text {opt }}\left(Q=0.01 I_{n}\right)$ & $\rho\left(Q=0.1 I_{n}\right)$ & $J_{\text {opt }}\left(Q=0.1 I_{n}\right)$ \\
\hline open loop & $1.0243 \mathrm{e}+004$ & & $1.0243 \mathrm{e}+004$ & \\
$C S F$ & $5.3867 \mathrm{e}-002$ & $6.441 \mathrm{e}+001$ & $9.94 \mathrm{e}-003$ & $3.215 \mathrm{e}+002$ \\
$A C S F$ & $5.5473 \mathrm{e}-002$ & $6.861 \mathrm{e}+001$ & $8.72 \mathrm{e}-007$ & $2.50 \mathrm{e}+002$ \\
\hline
\end{tabular}
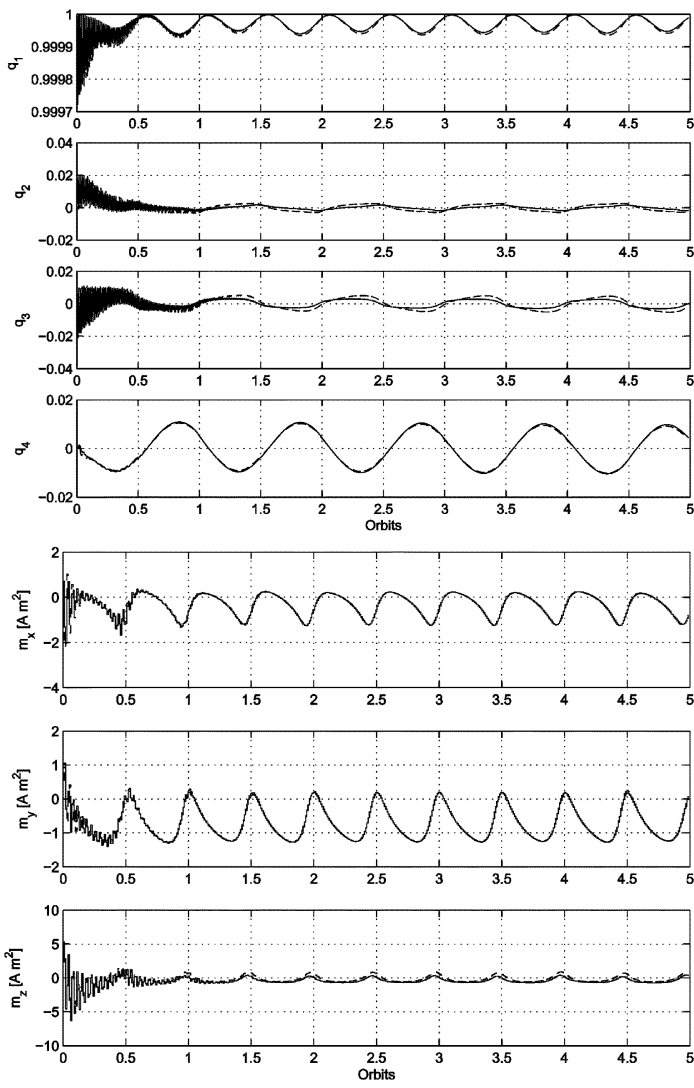

(a)
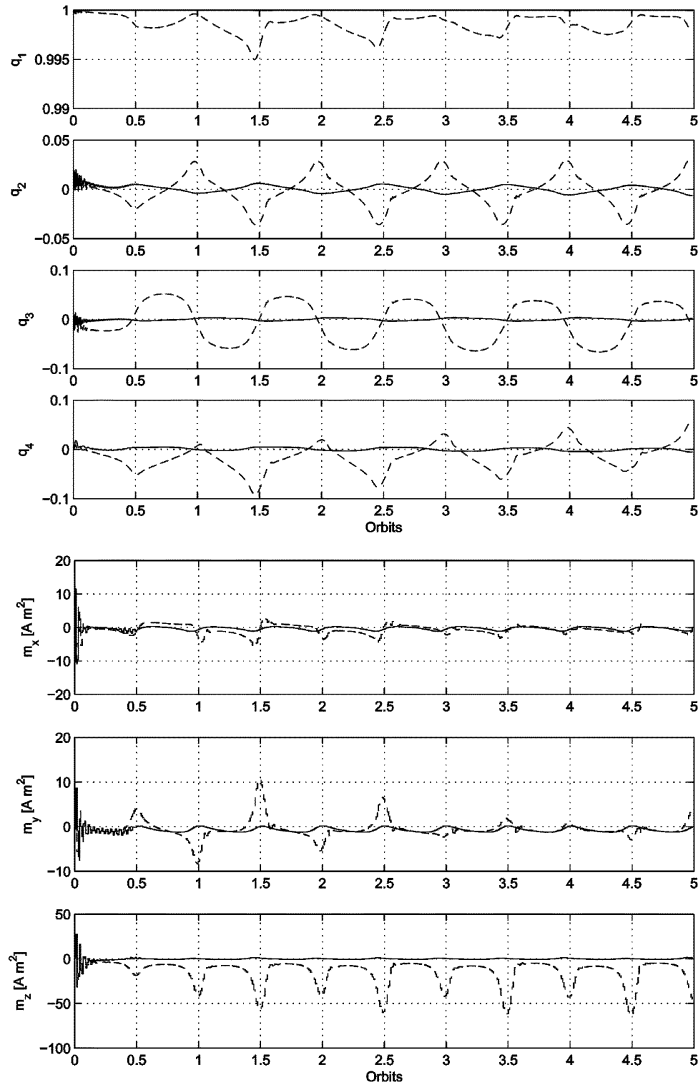

(b)

Fig. 2. Quaternion and control dipole moments for the CSF (solid lines) and ACSF (dashed lines) controllers. Left: $Q=0.01 I_{n}$. Right: $Q=0.1 I_{n}$.

able range for the control variables). The different behavior of the DOF1 controller can be easily interpreted by recalling that this controller replaces actual angular rate measurements with pseudo-derivatives computed on the basis of attitude measurements only: this leads to a higher equivalent derivative gain.

\section{E. Comparison With Averaging-Based Design}

Finally, in order to evaluate the benefits of the proposed procedure, which allows to take into account the actual periodicity of the linearized model in the design process, a comparison between the CSF controller and a similar controller tuned on the basis of a time-averaged linearized model (and denoted in the following with the acronym ACSF) has been performed (see also [26] for a more detailed comparison study on the magnetic control of roll/yaw dynamics for momentum bias spacecraft). More precisely, the averaging-based design has been performed as follows.

- The periodic linear model given by (1)-(2) and (15) has been averaged over one orbital period in order to obtain a time-invariant approximation. The averaged model has been then discretized, consistently with the corresponding periodic model $(N=100$ has been considered in the comparison).

- The discrete-time averaged model has been used to design an optimal state feedback gain. In order to ensure the consistency of the comparison, the design has been performed by minimizing the cost function (16). Furthermore, the design has been carried out for two different choices for the $Q$ weighting matrix, namely $Q=0.01 I_{n}$ and $Q=0.1 I_{n}$, while $R=100 I_{3}$.

The obtained results are presented in Table II and Fig. 2. As can be seen from the table, the two designed controllers lead to very similar results for the case of $Q=0.01 I_{n}$. This is reflected by the simulation results depicted in the left portion of the figure, which shows that similar closed-loop behavior is obtained in both cases. This result is not surprising: it is apparent from the figure that this choice of weights leads to a closed-loop settling time for the attitude error of about one orbit, so the effect of averaging in this case is not too significant. Significant discrepancies appear, on the other hand, when considering the 
case of $Q=0.1 I_{n}$ : in this case the weighting leads to significantly faster closed-loop dynamics (the transients in the right portion of the Figure settle in about one quarter of an orbit) so we expect the averaging-based controller to provide less satisfactory performance, which is indeed the case. Therefore, the design approach based on the periodic model proves superior whenever a high performance attitude control system, achieving fast (settling time shorter than one orbit) closed-loop dynamics is desired.

\section{CONCLUSION}

The problem of designing discrete-time attitude controllers for magnetically actuated spacecraft has been considered. An approach to the tuning of various classes of "projection based" controllers has been proposed, relying on periodic optimal output feedback control techniques. The performances of the proposed control algorithms have been discussed and illustrated in a detailed simulation study.

All the considered control designs have provided highly satisfactorily performance, and proved the capability to overcome one or both main restrictions posed by the reference periodic optimal state feedback control design, i.e., demanding memory storage requirements and full state measurements availability. Finally, the improved performance with respect to methods based on averaged models has been demonstrated.

\section{REFERENCES}

[1] A. C. Stickler and K. T. Alfriend, "An elementary magnetic attitude control system,” J. Spacecraft Rockets, vol. 13, no. 5, pp. 282-287, 1976.

[2] H. Hablani, "Comparative stability analysis and performance of magnetic controllers for bias momentum satellites," J. Guid., Control Dyn., vol. 18, no. 6, pp. 1313-1320, 1995.

[3] M. Pittelkau, "Optimal periodic control for spacecraft pointing and attitude determination," J. Guid., Control Dyn., vol. 16, no. 6, pp. 1078-1084, 1993.

[4] A. Varga and S. Pieters, "Gradient-based approach to solve optimal periodic output feedback control problems," Automatica, vol. 34, no. 4, pp. 477-481, 1998.

[5] R. Wisniewski and L. M. Markley, "Optimal magnetic attitude control," presented at the 14th IFAC World Congr., Beijing, China, 1999.

[6] M. Lovera, E. De Marchi, and S. Bittanti, "Periodic attitude control techniques for small satellites with magnetic actuators," IEEE Trans. Control Syst. Technol., vol. 10, no. 1, pp. 90-95, Jan. 2002.
[7] M. Lovera, "Optimal magnetic momentum control for inertially pointing spacecraft," Eur. J. Control, vol. 7, no. 1, pp. 30-39, 2001.

[8] M. Psiaki, "Magnetic torquer attitude control via asymptotic periodic linear quadratic regulation," J. Guid., Control Dyn., vol. 24, no. 2, pp. 386-394, 2001.

[9] E. Silani and M. Lovera, "Magnetic spacecraft attitude control: A survey and some new results," Control Eng. Practice, vol. 13, no. 3, pp. 357-371, 2005.

[10] H. Yan, M. Ross, and K. Alfriend, "Three-axis magnetic attitude control using pseudospectral control law," presented at the AAS/AIAA Astrodyn. Specialists Conf., Lake Tahoe, 2005, Paper AAS 05-417.

[11] M. Wood, W.-H. Chen, and D. Fertin, "Model predictive control of low earth orbiting spacecraft with magneto-torquers," presented at the IEEE Int. Conf. Control Appl., Munich, Germany, 2006.

[12] F. Martel, P. K. Pal, and M. Psiaki, "Active magnetic control system for gravity gradient stabilised spacecraft," presented at the 2nd Ann. AIAA/USU Conf. Small Satellites, Logan, UT, 1988.

[13] A. Varga, "A periodic systems toolbox for Matlab," presented at the 16th IFAC World Congr., Prague, Czech Republic, 2005.

[14] M. Sidi, Spacecraft Dynamics and Control. Cambridge, U.K.: Cambridge University Press, 1997.

[15] P. Hughes, Spacecraft Attitude Dynamics. New York: Wiley, 1986.

[16] R. Wisniewski and J. Stoustrup, "Periodic $H_{2}$ synthesis for spacecraft attitude determination and control with a vector magnetometer and magnetorquers," in Proc. 1st IFAC Workshop Periodic Control Syst., , Cernobbio-Como, Italy, 2002, pp. 121-126.

[17] S. Bittanti and P. Colaneri, Periodic Systems: Filtering and Control. : Springer, 2008.

[18] A. Varga, "Periodic Lyapunov equations: Some applications and new algorithms," Int. J. Control, vol. 67, no. 1, pp. 69-87, 1997.

[19] M. Lovera and A. Astolfi, "Spacecraft attitude control using magnetic actuators," Automatica, vol. 40, no. 8, pp. 1405-1414, 2004.

[20] M. Lovera and A. Astolfi, "Global magnetic attitude control of spacecraft in the presence of gravity gradient," IEEE Trans. Aerosp. Electron. Syst., vol. 42, no. 3, pp. 796-805, Jul. 2006.

[21] C. Farges, D. Peaucelle, and D. Arzelier, "LMI formulation for the resilient dynamic output feedback stabilization of linear periodic systems," presented at the 13th IFAC Workshop Control Appl. Opt., Paris, France, 2006.

[22] M. Lovera, "Control-oriented modelling and simulation of spacecraft attitude and orbit dynamics," J. Math. Comput. Model. Dyn. Syst., vol. 12 , no. 1 , pp. 73-88, 2006.

[23] P. Fritzson and Bunus, "Modelica-A general object-oriented language for continuous and discrete-event system modelling and simulation," in Proc. 35th IEEE Ann. Simulation Symp., 2002, pp. 365-380.

[24] O. Montenbruck and E. Gill, Satellite Orbits: Models, Methods, Applications. New York: Springer, 2000.

[25] J. Wertz, Spacecraft Attitude Determination and Control. Dordrecht, Holland: D. Reidel Publishing Company, 1978.

[26] T. Pulecchi, M. Lovera, and A. Varga, "Classical vs modern magnetic attitude control design: A case study," presented at the 7th Int. ESA Conf. Guid., Nav. Control Syst., Tralee, Ireland, 2008. 